\title{
Gate-Controlled Supercurrent in Epitaxial Al/InAs Nanowires
}

\author{
Tosson Elalaily, Olivér Kürtössy, Zoltán Scherübl, Martin Berke, Gergö Fülöp, István Endre Lukács, \\ Thomas Kanne, Jesper Nygård, Kenji Watanabe, Takashi Taniguchi, Péter Makk,* and Szabolcs Csonka*
}

Cite This: Nano Lett. 2021, 21, 9684-9690

Read Online

ACCESS I

山lll Metrics \& More

回国 Article Recommendations

S1 Supporting Information

ABSTRACT: Gate-controlled supercurrent (GCS) in superconducting nanobridges has recently attracted attention as a means to create superconducting switches. Despite the clear advantages for applications, the microscopic mechanism of this effect is still under debate. In this work, we realize GCS for the first time in a highly crystalline superconductor epitaxially grown on an InAs nanowire. We show that the supercurrent in the epitaxial Al layer can be switched to the normal state by applying $\simeq \pm 23 \mathrm{~V}$ on a bottom gate insulated from the nanowire by a crystalline $\mathrm{hBN}$ layer. Our

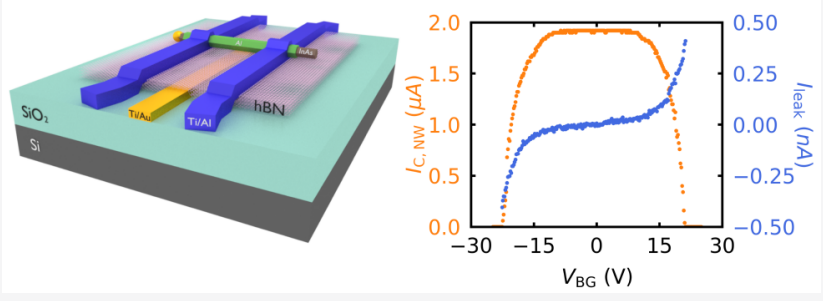
extensive study of the temperature and magnetic field dependencies suggests that the electric field is unlikely to be the origin of GCS in our device. Though hot electron injection alone cannot explain our experimental findings, a very recent non-equilibrium phonons based picture is compatible with most of our results.

KEYWORDS: field effect, epitaxial superconductors, nanowire, gate-controlled supercurrent, hot electron injection, phonons

\section{INTRODUCTION}

Superconducting circuits have become promising building blocks in various architectures for quantum computing devices, ${ }^{1,2}$ single photon detectors, ${ }^{3,4}$ quantum-limited amplifiers, ${ }^{5}$ phase-coherent caloritronics, ${ }^{6,7}$ ultrasensitive magnetometers $^{8,9}$ and fast classical supercomputers. ${ }^{10,11}$ In the latter, the superconducting electronics are integrated with semiconductor technology. In particular, rapid single flux quantum (RSFQ) devices become more desirable than semiconducting switches due to their fast switching speed and low power consumption. ${ }^{10}$ Since RSFQ consists of a flux-controlled superconducting loop with Josephson junctions along with onchip coils, its upscaling remains a challenge. In order to realize scalable superconducting switches, electrical control via gate electrodes would be desirable.

Very recently a striking new effect was observed in metallic nanostructures. In superconducting nanobridges the supercurrent can be controlled by applying a voltage on a closely spaced gate electrode. ${ }^{12-24}$ By increasing the gate voltage beyond a certain threshold, the supercurrent in the nanobridge can be quenched. Previous works show GCS in thin metallic nanowires, ${ }^{12,18,22,23}$ in proximitized normal metal in a superconductor-normal-superconductor (SNS) junction ${ }^{13}$ and in Dayem nanobridges. ${ }^{15,17,20}$ In addition to high-speed superconducting switches, ${ }^{23}$ observation of GCS in superconducting nanostructures led to the realization of new nanodevices such as gate-controlled superconducting phase shifter ${ }^{16}$ and halfwave nanorectifiers. ${ }^{20}$ Despite the clear advantages of GCS for applications, the origin of the effect is still under debate. Some works explained the suppression of the supercurrent by an electric field induced perturbation of the superconducting state, ${ }^{25-27}$ such as, e.g., by Swinger effect, ${ }^{28}$ others attributed the effect to injection of high-energy quasiparticles tunneling from the gate electrode. ${ }^{22-24}$ Even though the microscopic origin of the effect is unclear, gate-controlled nanobridges can work at ultralow power and high switching speed. In addition, their configuration can be easily scaled up, which provides a promising building block for superconducting switches in modern architectures of both classical and quantum computers.

Recently InAs semiconductor nanowires with epitaxial $\mathrm{Al}$ superconducting shells ${ }^{29}$ have become the primary platform for research on various potential quantum devices. Concepts have been developed for topologically protected qubits ${ }^{30}$ and surface code for majorana states ${ }^{31}$ based on hybrid nanowires, and they are also promising for realization of gatemon or Andreev qubits. ${ }^{32,33}$ In all of these examples of quantum hardware, the gate tunable superconductivity would be highly desirable to provide an additional experimental control knob of the system. However, up to now, all gate tuning experiments were performed on polycrystalline materials and therefore it has not been clear if GCS exists for highly crystalline materials. In this work, we studied for the first time the superconducting gating effect in highly crystalline $\mathrm{Al}$ shells epitaxially grown on

Received: September 8, 2021

Revised: October 20, 2021

Published: November 2, 2021 
InAs nanowires. ${ }^{29}$ We will show in the following that the superconducting state can be switched off by applying voltage on a nearby metallic electrode and provide detailed characteristics of the gating behavior.

\section{RESULTS AND DISCUSSION}

We have used InAs nanowires grown by molecular beam epitaxy (MBE) using gold nanoparticles as catalysts. After InAs nanowires growth, an $\mathrm{Al}$ shell layer of thickness $20 \mathrm{~nm}$ was epitaxially grown by deposition within the MBE chamber at low temperature. By rotating the substrate during $\mathrm{Al}$ growth, the $\mathrm{Al}$ shell layer is grown in all InAs nanowire facets, resulting in fully covered nanowires. ${ }^{29}$

To investigate the gate-controlled supercurrent in InAs nanowires with epitaxial superconducting layer, first, we fabricated devices with the standard geometry using side gates $^{12-23}$ or a backagte, ${ }^{12}$ where the gating effect was observed in eight devices. In order to improve the geometry, i.e., minimize the separation between the gate and the nanowire and keep the leakage current small, we developed the geometry presented in Figure $1 \mathrm{a}-\mathrm{c}$. A metallic gate from $\mathrm{Ti} / \mathrm{Au}$ (yellow) with thicknesses of $7 / 33 \mathrm{~nm}$ was fabricated on an intrinsic Si wafer with a $290 \mathrm{~nm}$ thick oxide layer. To insulate the gate from the wire, 20-30 nm thick hBN (pink) was stacked on the gate electrode with PDMS-based dry transfer technique. $\mathrm{hBN}$ is an excellent single crystal insulator between the gate and the wire, serving as a tunnel barrier. ${ }^{34-38}$ The nanowire (gray) with $\mathrm{Al}$ shell (green) was deposited by a micromanipulator on top of the hBN layer. Two pairs of $\mathrm{Al}$ contacts (blue) have been fabricated on the top of the nanowire with a distance of $1.5 \mu \mathrm{m}$ to allow quasi-four-probe measurements. More details about fabrication are given in Methods (see the Supporting Information).

The current-voltage $(I-V)$ characteristics of the nanowire device measured at $40 \mathrm{mK}$ clearly show a well developed zero resistance state (see green curve in Figure 1d) corresponding to a supercurrent flowing through the $\mathrm{Al}$ shell of the wire. Two clear switches to a finite resistance state are observed at 1.94 and $2.34 \mu \mathrm{A}$. Similar multiple transitions were observed in a suspended $\mathrm{Ti}$ nanowire. ${ }^{18}$ The nanowire device shows a hysteretic behavior and switches back at two successive retrapping current values at $I_{\mathrm{r}, 1}=1.94 \mu \mathrm{A}$ and $I_{\mathrm{r}, 2}=1.74 \mu \mathrm{A}$ when the measurements were carried out in the opposite ramping direction. To identify the origin of the two switching steps, we have separately measured the $I-V$ curves for each horizontal pair of contacts (blue electrodes in Figure 1a) using a two-probe method. The measurements for the top pair with blue curve switch at $2.34 \mu \mathrm{A}$, while the bottom electrodes switch at $7.5 \mu \mathrm{A}$ (see the Supporting Information). From this, we could attribute the switching at the lower current to the SCnormal transition of the epitaxial $\mathrm{Al}$ shell, $I_{\mathrm{C}, \mathrm{NW}}$, while the switching at the higher current is the same as that of the $\mathrm{Al}$ contact segment above the nanowire, $I_{\mathrm{C}, \mathrm{C}}$, which is marked by the red rectangle in Figure 1a.

The dependence of the supercurrent on the gate voltage was investigated by measuring the $I-V$ curve of the nanowire device as a function of the bottom gate voltage, $V_{\mathrm{BG}}$ (see Figure $2 \mathrm{a}$ ). The white regions represent the zero resistance state. With increasing $V_{\mathrm{BG}}$ with either negative or positive polarity, both $I_{\mathrm{C}, \mathrm{NW}}$ and $I_{\mathrm{C}, \mathrm{C}}$ remain constant. Beyond the threshold at $V_{\mathrm{BG}} \simeq \pm 12 \mathrm{~V}$, both critical currents are suppressed together up to full suppression at the critical gate voltage, $V_{\mathrm{BGC}}$ $\simeq \pm 23 \mathrm{~V}$, at which the device is switched to the normal state.
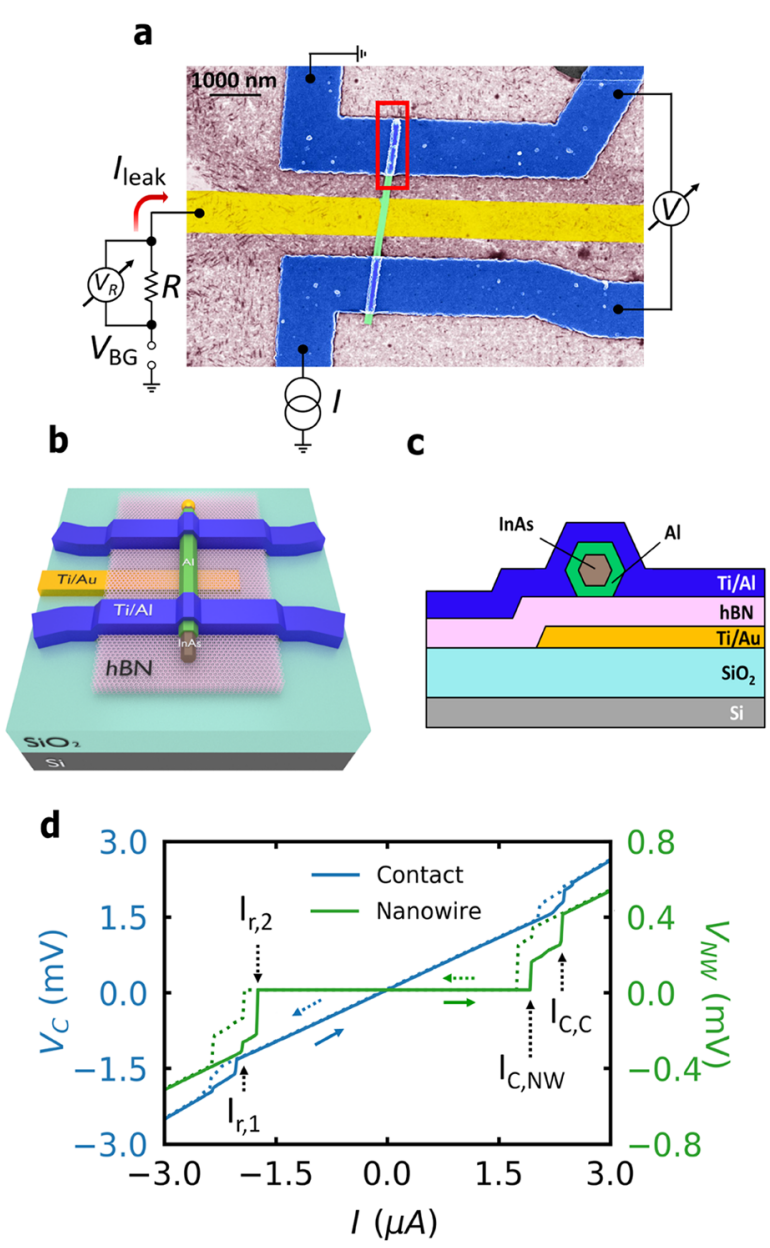

Figure 1. Device configuration. (a) False colored SEM image of the fabricated device with schematics of the device circuit. Schematic of the device with (b) $45^{\circ}$ angle view and (c) side view. (d) $I-V$ characteristics of the nanowire device (green curve) at $40 \mathrm{mK}$ with two different switchings at $I_{\mathrm{C}, \mathrm{NW}}$ and $I_{\mathrm{C}, \mathrm{C}}$ in the case of bias current is ramped from negative to positive values (solid green arrow). In the opposite ramping direction (dotted green arrow), it switches back at two successive retrapping current values, $I_{\mathrm{r}, 1}$ and $I_{\mathrm{r}, 2}$. Measurement of the top pair of $\mathrm{Al}$ contacts using the two-probe method (blue curve), showing the switching of the contacts at the same value of $I_{\mathrm{C}, \mathrm{C}}$.

The maximum electric field estimated at the critical gate voltage is $E_{\max } \approx 200 \mathrm{MVm}^{-1}$, which is in the same order of magnitude as that reported in refs 18 and 39. The fine characteristics of the $I-V$ curves are better visible in Figure $2 b$, where the red and gray dashed lines trace the suppression of $I_{\mathrm{C}, \mathrm{NW}}$ and $I_{\mathrm{C}, \mathrm{C}}$ with increasing $V_{\mathrm{BG}}$, respectively. We note that with increasing $V_{\mathrm{BG}}$, the difference between the retrapping current $I_{\mathrm{r}, 2}$ and $I_{\mathrm{C}, \mathrm{C}}$ is decreased and fully vanished at $V_{\mathrm{BG}} \approx$ $\pm 22 \mathrm{~V}$

The leakage current, $I_{\text {leak, }}$ from the gate to the nanowire device was also measured within a $\pm V_{\mathrm{BGC}}$ window by recording the voltage across the pre-resistor on the gate (see Figure 1a). The leakage current was then corrected by subtracting the leakage between lines of the cryostat (see Supporting Information). ${ }^{23}$ The measured $I_{\text {leak }}$ shows an exponential increase with $V_{\mathrm{BG}}$ for both polarities (see Figure 2c), noting that, a strong increase is seen in the leakage current around the gate voltages, where the supercurrents get suppressed.

The critical temperature was determined by measuring the $I-V$ characteristics of the device at different elevated 
a
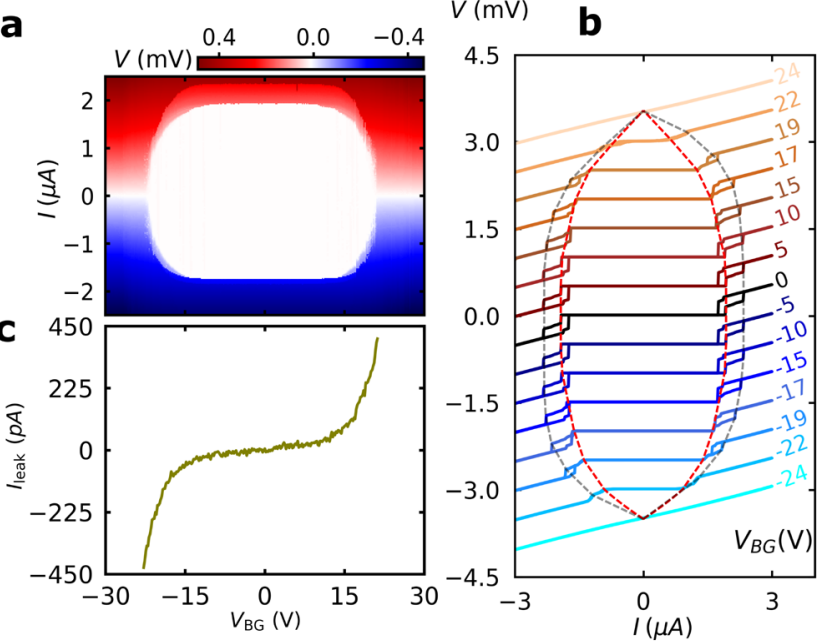

Figure 2. Gating of supercurrent. (a) $I-V$ characteristics of the nanowire device as a function of bipolar voltage applied to the bottom gate, $V_{\mathrm{BG}}$, as the current was sweeping from negative to positive values. (b) High-resolution $I-V$ curves measured at selected gate voltages and equally separated on $y$-axis for better visibility. The red and gray dashed lines trace the suppression of $I_{\mathrm{C}, \mathrm{NW}}$ and $I_{\mathrm{C}, \mathrm{C}}$ with increasing $V_{\mathrm{BG}}$, respectively. (c) Measured and corrected leakage current from bottom gate to nanowire device as a function of $V_{\mathrm{BG}}$.

temperatures at $V_{\mathrm{BG}}=0$ (see Figure $3 \mathrm{a}$ ). In the case of $I_{\mathrm{C}, \mathrm{NW}}$, it is fully quenched at $T_{\mathrm{C}, \mathrm{NW}} \simeq 1050 \mathrm{mK}$, while for $I_{\mathrm{C}, \mathrm{C}}$ at $T_{\mathrm{C}, \mathrm{C}} \simeq$ $1400 \mathrm{mK}$ with corresponding normal state resistances $R_{\mathrm{n}, \mathrm{NW}}=$ $135 \Omega$ and $R_{\mathrm{n}, \mathrm{C}}=191 \Omega$, respectively. By extracting the values of $I_{\mathrm{C}, \mathrm{NW}}$ and $I_{\mathrm{C}, \mathrm{C}}$, the dependence of the critical currents on temperature is plotted in Figure $3 \mathrm{~b}$. The red dashed-dotted and gray dotted curves are fits of the temperature dependences of $I_{\mathrm{C}, \mathrm{NW}}$ and $I_{\mathrm{C}, \mathrm{C}, 42}$, respectively, by using the AmbegaokarBaratoff relation: ${ }^{0-42}$

$$
I_{\mathrm{C}} R_{\mathrm{n}}=\frac{\pi}{2 e} \Delta(T) \tanh \left(\frac{\Delta(T)}{2 \mathrm{k}_{\mathrm{B}} T}\right)
$$

where

$$
\Delta(T)=\Delta(0) \tanh \left(a \sqrt{\frac{T_{\mathrm{C}}}{T}-1}\right)
$$

is the superconducting gap at temperature $T,{ }^{43-45} R_{\mathrm{n}}$ is the normal-state resistance, and $k_{\mathrm{B}}$ is Boltzmann constant. The temperature dependences of both $I_{\mathrm{C}, \mathrm{NW}}$ and $I_{\mathrm{C}, \mathrm{C}}$ are fitted using the coefficient $a=2$ and 2.4 and $R_{\mathrm{n}}=130$ and $143 \Omega$, respectively. The latter values of the normal-state resistances are in good agreement with our experimental findings.

The temperature dependence of GCS has been investigated by measuring the critical currents, $I_{\mathrm{C}, \mathrm{NW}}$ and $I_{\mathrm{C}, \mathrm{C}}$ as a function of bipolar gate voltage at elevated temperatures (see Figure $3 \mathrm{c}, \mathrm{d}$, respectively). With increasing of the bath temperature, both $I_{\mathrm{C}, \mathrm{NW}}$ and $I_{\mathrm{C}, \mathrm{C}}$ at zero gate voltage respect the temperature dependence of critical currents shown in Figure $3 \mathrm{~b}$. However, the gating characteristics look quite similar at all temperatures. The critical gate voltage, $V_{\mathrm{BGC}}$, in the case of $I_{\mathrm{C}, \mathrm{NW}}$ did not change with increasing $T$ up to close to its critical temperature at $T_{\mathrm{C}, \mathrm{NW}}$, while in the case of $I_{\mathrm{C}, \mathrm{C}}$, it only shifts to lower values (indicated by red arrow) for measurements at temperatures higher than $T_{\mathrm{C}, \mathrm{NW}}$. A similar shift of $V_{\mathrm{BGC}}$ with increasing temperature was observed in ref 18 . We have also plotted the a

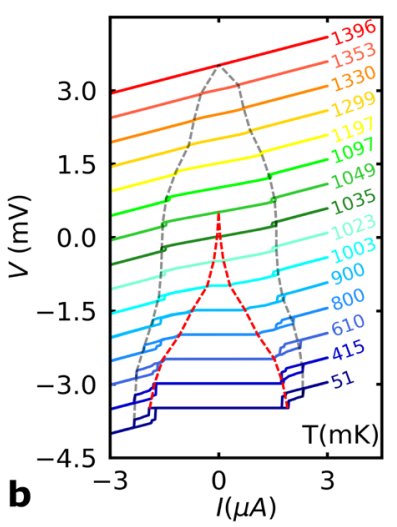

C
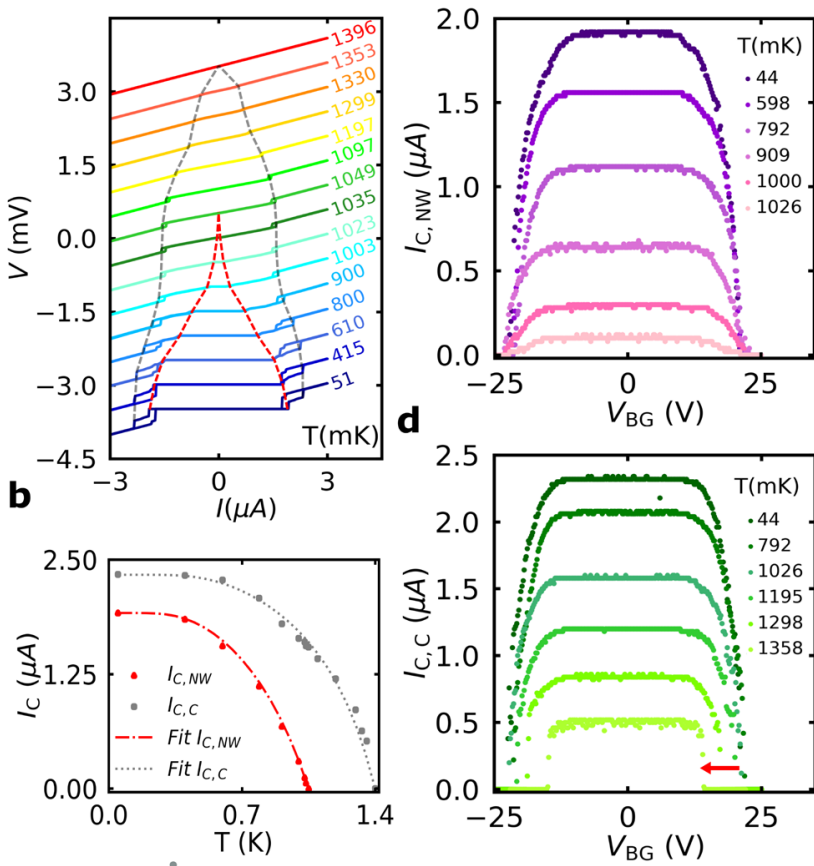

Figure 3. Temperature dependence. (a) $I-V$ characteristics of the nanowire device at elevated temperatures up to $1400 \mathrm{mK}$ and equally separated on $y$-axis for better visibility. Red and gray dashed lines trace the suppression of both $I_{\mathrm{C}, \mathrm{NW}}$ and $I_{\mathrm{C}, \mathrm{C}}$ with increasing temperature, respectively. $I_{\mathrm{C}, \mathrm{NW}}$ is fully quenched at critical temperature $T_{\mathrm{C}, \mathrm{NW}} \simeq 1050 \mathrm{mK}$, while $I_{\mathrm{C}, \mathrm{C}}$ at $T_{\mathrm{C}, \mathrm{C}} \simeq 1400 \mathrm{mK}$. (b) Temperature dependence of $I_{\mathrm{C}, \mathrm{NW}}$ and $I_{\mathrm{C}, \mathrm{C}}$ extracted from panel a. Temperature dependence of the critical currents is fitted by using Ambegaokar-Baratoff relation illustrated by the red dashed-dotted and the gray dotted lines for $I_{\mathrm{C}, \mathrm{NW}}$ and $I_{\mathrm{C}, \mathrm{C}}$, respectively. (c, d) Critical current as a function of bipolar gate voltage for both $I_{\mathrm{C}, \mathrm{NW}}$ and $I_{\mathrm{C}, \mathrm{C}}$ at elevated temperatures up to $98 \%$ of their critical temperatures, respectively.

critical currents as a function of leakage current for different temperatures (see the Supporting Information). A small change in the critical leakage current is visible due to fluctuations in the leakage current.

The dependence of critical currents with magnetic field was investigated by measuring $I-V$ characteristics of the nanowire device as a function of out of plane magnetic field, $B$, as shown in Figure 4a. Both $I_{\mathrm{C}, \mathrm{NW}}$ and $I_{\mathrm{C}, \mathrm{C}}$ decrease in the magnetic field, as expected. Moreover, it can be also seen that $I_{\mathrm{C}, \mathrm{NW}}$ and $I_{\mathrm{C}, \mathrm{C}}$ cross each other at $B \simeq \pm 24 \mathrm{mT}$, and their corresponding critical fields are $B_{\mathrm{C}, \mathrm{NW}}=66 \mathrm{mT}$ and $B_{\mathrm{C}, \mathrm{C}}=50 \mathrm{mT}$, respectively. This is clearly seen in Figure $4 \mathrm{~b}$, which shows the magnetic field dependence of critical currents extracted from measurements in Figure 4a.

The dependence of GCS in the nanowire device in finite magnetic field shows a similar dependence as its temperature dependence: that the gate dependence has the same general trend at all magnetic fields up to $46 \mathrm{mT}$ as shown in Figure $4 \mathrm{c}$ in the case of $I_{C, C}$. On the other hand, $V_{\mathrm{BGC}}$ decreases when $B$ values approach $B_{\mathrm{C}, \mathrm{C}}$. In the same way, $I_{\mathrm{C}, \mathrm{NW}}$ shows a similar magnetic field dependence (see the Supporting Information). We have also measured both critical currents, $I_{\mathrm{C}, \mathrm{NW}}$ and $I_{\mathrm{C}, \mathrm{C}}$, as a function of the magnetic field at different gate voltages up to values very close to $V_{\mathrm{BGC}}$. A significant shift in $B_{\mathrm{C}, \mathrm{NW}}$ and $B_{\mathrm{C}, \mathrm{C}}$ to smaller values is observed by increasing $V_{\mathrm{BG}}$ higher than 15 


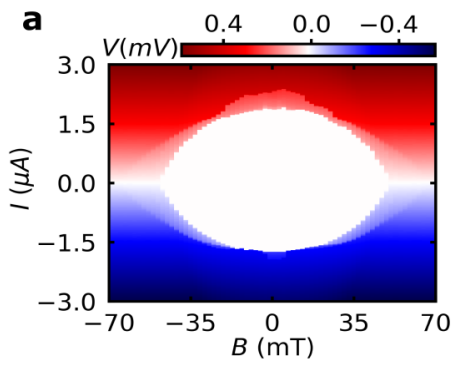

b

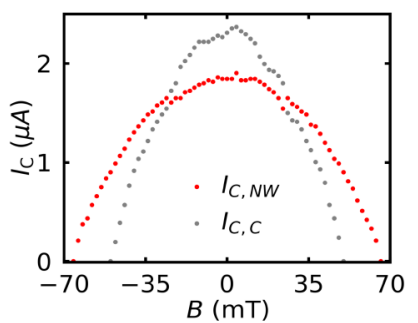

C

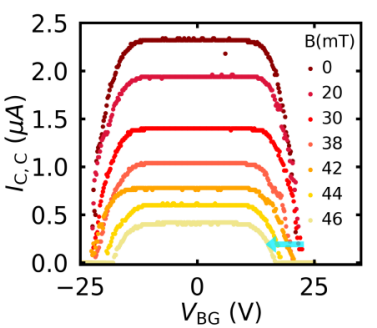

$\mathbf{f}$ d

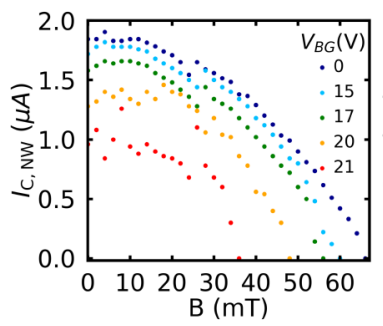

$\mathbf{e}$

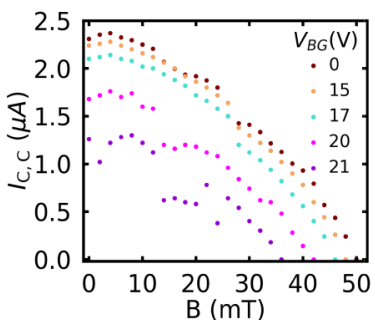

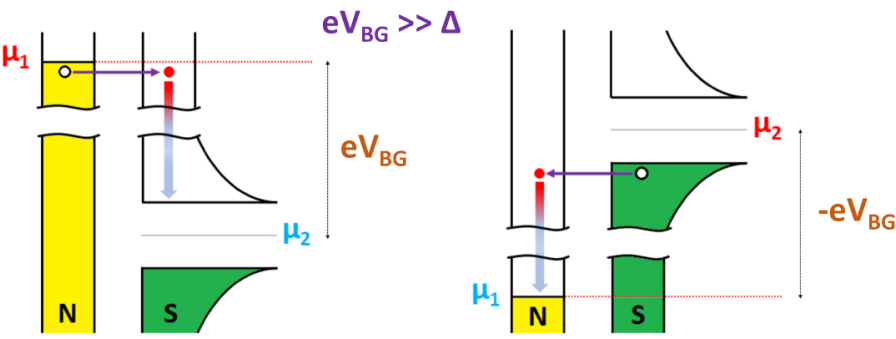

Figure 4. Magnetic field dependence and schematic of ballistic hot electron injection. (a) $I-V$ characteristics of the nanowire device as a function of out of plane magnetic field. (b) Critical currents of the nanowire device and the contact interface region as a function of the applied magnetic field, extracted from the measurements in panel a. $I_{\mathrm{C}, \mathrm{NW}}$ and $I_{\mathrm{C}, \mathrm{C}}$ cross each other at $B \simeq \pm 24 \mathrm{mT}$, leading to corresponding critical magnetic fields, $B_{\mathrm{C}, \mathrm{NW}}=66 \mathrm{mT}$ and $B_{\mathrm{C}, \mathrm{C}}=50 \mathrm{mT}$, respectively. (c) $I_{\mathrm{C}, \mathrm{C}}$ as a function of bipolar gate voltage at magnetic fields up to $46 \mathrm{mT}$. (d, e) $I_{\mathrm{C}, \mathrm{NW}}$ and $I_{\mathrm{C}, \mathrm{C}}$ as a function of magnetic field at gate voltage values close to the critical gate voltage, respectively. (f) Schematic diagrams of ballistic electron injection from/to the metallic gate $\mathrm{N}$ to/from the superconducting nanowire device $\mathrm{S}$ in the left and right panels, respectively. Colored/uncolored parts represent occupied/unoccupied states. As the hot electron (red circle) tunnels, it will relax to the lowest unoccupied state, releasing heat on either the $\mathrm{N}$ or $\mathrm{S}$ side, resulting in different heating of the $\mathrm{S}$ side as the polarity of $V_{\mathrm{BG}}$ changes.

$\mathrm{V}$ as illustrated in Figure 4d,e, respectively. Similar dependence with $B$ field was observed in Ti-based superconducting nanostructures. $^{12,15} \mathrm{We}$ also note that a large scattering in the critical currents values was observed in the measurements at $V_{\mathrm{BG}}>17 \mathrm{~V}$, which can be attributed to the wide nonthermal switching current distribution as a result of the gating effect. ${ }^{19,24,39,46}$

In previous studies, the origin of GCS was attributed to two different mechanisms, either to the effect of the applied electric field $^{12-21,25-28}$ or to high-energy quasiparticle injection via tunneling. $^{22-24}$ We will compare our experimental findings with these explanations in the following.

GCS has been reported in various device geometries of evaporated polycrystalline metallic nanobridges made of different superconducting materials, ${ }^{12-21}$ and an explanation based on electric field induced distortion of the superconducting wave function that could destroy the BCS state has been proposed, ${ }^{25-27}$ such as, e.g., by Swinger effect. ${ }^{28}$ The observed $B$ field and $T$ dependencies of the gating effect show characteristics very similar to our epitaxial superconductor case. However, there is a finite leakage current of $\approx 100 \mathrm{pA}$ at gate voltages where the supercurrent is reduced (see Figure $2 \mathrm{a}, \mathrm{c})$. This leakage current is largely $B$-field and temperature independent, as expected (see the Supporting Information). Assuming that the leakage takes place between the gate electrode and the nanowire, in the simplest ballistic picture, hot electrons are injected into the superconducting shell with energies even as high as $10-20 \mathrm{eV}$, which is several orders of magnitude higher than the SC gap. These electrons could heat up the superconducting bridge and drive it to the normal state, as it is proposed by refs $22-24$ as a microscopic origin of the gating effect. Our basic estimation (see the Supporting Information) of induced heat transfer also suggests that the hot electrons could bring the temperature of the epitaxial shell in the range of the superconducting critical temperature. Instead of using silicon dioxide or other amorphous insulators, the gate electrode and the superconductor are separated by a 20-30 $\mathrm{nm}$ thick single crystalline $\mathrm{hBN}$ layer in our device, which is a large band gap insulator commonly used as a tunnel barrier in $2 \mathrm{D}$ electronics. ${ }^{34,35,38}$ Considering a tunnel barrier between the gate and superconductor, the heating effect resulting from hot electron injection should show a strong asymmetric dependence on the polarity of the gate voltage. For the polarity when electrons tunnel from the gate electrode to the superconductor (see Figure $4 \mathrm{f}$ (left)), hot electrons relax their energy in the superconductor by inducing a large number of quasiparticles, which results in a significant heat load. On the other hand, for opposite polarity (see Figure 4f (right)), hot electrons heat the metal block of a large gate electrode, which has a much smaller heating effect on the superconductor isolated by the gate insulator. Such gate voltage asymmetry was observed in ref 22 (see Figure 6 in the extended data for the reference). However, it does not appear in our measurements (see Figure 2a) after the initial training period (see the Supporting Information), which contradicts the simple explanation based on ballistic injection of hot electrons. Nevertheless, we should also consider within this comparison the difference in the device geometry between the investigated device (in ref 22) and the device presented here in our work.

However, if the tunneling process is not ballistic through the $\mathrm{hBN}$, inelastic processes could lead to dissipation within the barrier itself, resulting in more symmetric $I-V$ curves. These inelastic excitations could most likely be phonons, as explained at the end of this section. However, in suspended nanobridges presented in ref 18 , the leakage current and the generation of inelastic excitations are suppressed by orders of magnitude, so the gating effect in these devices could have another origin besides the injection of hot electrons/phonons. 
Considering the magnetic field dependence at finite gate voltage (Figure 4d,e), it is consistent with hot electron injection, since increasing the gate voltage leads to an increase in the energy and rate of high-energy electrons injected into the nanowire segment. As a result, the device heats up more and the critical field of the superconducting wire decreases to smaller values. Moreover, as the magnetic field increases, the critical temperature of the superconductor decreases. In turn, a smaller gate voltage could bring the electronic temperature of the device up to this reduced critical temperature of the superconductor. This is visible in Figure 4c, where the reduction of the critical gate voltage is shown.

Finally, we focus on the $T$ dependence of $V_{\mathrm{BGC}}$. In a simple hot electron injection scenario, one would expect that at elevated temperatures, a lower heat load would be sufficient to drive the contact segment to normal state. However, our results show that $V_{\mathrm{BGC}}$ for the nanowire does not depend on the temperature up to $T_{\mathrm{C}, \mathrm{NW}}$. This alone would be consistent with an electric field-based origin. At temperatures larger than $T_{\mathrm{C}, \mathrm{NW}}$, the nanowire is in the normal state and only the contacts are superconducting. As shown by the red arrow on Figure $3 \mathrm{~d}, V_{\mathrm{BGC}}$ decreases at higher temperatures, as expected from Joule heating scenarios. We note that above $T_{\mathrm{C}, \mathrm{NW}}$ the wire itself is already resistive, thus current flow induces additional dissipation, which could also contribute.

In summary, the temperature dependence cannot be described by a simple ballistic injection of hot electrons alone, but the strong dependence on the leakage current also makes the origin due to an electric field unlikely.

At the end we note a parallel work of Ritter et al., ${ }^{39}$ in which the authors investigated the origin of the gating effect on TiN nanowires. They found that the decrease in supercurrent for their devices was independent of the electric field between the wire and the gate electrode. The suppression of the supercurrent was attributed to the generation of nonequilibrium phonons due to relaxation of high-energy electrons in the substrate. The generated phonons could propagate through the substrate over distances that exceed $1 \mu \mathrm{m}$. Once these high-energy phonons reach to the superconducting device, they generate a large number of quasiparticles and suppress the supercurrent. A similar scenario is likely to be present in our device, where the phonons spread through the $\mathrm{hBN}$ layer and suppress the supercurrent in the nanowire and contact segments. Moreover, this scenario explains, for example, the symmetry of GCS with gate voltage present in our measurements. The observed magnetic field and temperature dependencies are also compatible with heat transfer via phonons.

To sum up, the phonon generation scenario might explain most of our findings; however, it is likely that the origin of the GCS effect depends heavily on the device architecture, since, e.g., for suspended devices of ref 18 , another origin might be present. In order to achieve reliable, fast, and integrated superconducting electronics based on GCS, further detailed studies on the various existing experimental platforms are required.

\section{CONCLUSIONS}

In summary, we have demonstrated the superconducting gating effect in an epitaxially grown superconducting layer for the first time. We developed a novel gate-controlled supercurrent transistor using an $\mathrm{Al}$ shell around an InAs nanowire, which acts as the active region. The device shows a full suppression of the supercurrent by applying $\simeq \pm 23 \mathrm{~V}$ on a bottom gate insulated from the nanowire by a high-quality single crystalline $\mathrm{hBN}$ layer. Detailed magnetic field and temperature dependent characterization allowed us to compare the experimental facts with existing scenarios of superconducting gating. The gating effect independent of the polarity suggests that the simple ballistic hot electron injection does not provide a complete explanation of the observed gating. However, the strong correlation between the suppression of the critical current and the increase in the leakage current suggests that the electric field is unlikely an origin of the gating effect in our particular device. Phonon generation in the tunnel barriers, however, can give an explanation which is consistent with most of our findings. Besides the fundamental interest, our results open the way to integrate superconducting switches into novel $\mathrm{Al} / \mathrm{InAs}$-based hybrid quantum architectures.

\section{ASSOCIATED CONTENT}

\section{(s) Supporting Information}

The Supporting Information is available free of charge at https://pubs.acs.org/doi/10.1021/acs.nanolett.1c03493.

Methods used in device fabrication and experiments, measurement of the other pair of the contacts, correction of the leakage current, correlation between the leakage current and the critical current, magnetic field dependence of the nanowire segment, influence of temperature and magnetic field on the leakage current, theoretical model, and initial training measurements (PDF)

\section{AUTHOR INFORMATION}

\section{Corresponding Authors}

Péter Makk - Department of Physics and Nanoelectronics "Momentum" Research Group of the Hungarian Academy of Sciences, Budapest University of Technology and Economics, 1111 Budapest, Hungary; (ㅇ) orcid.org/0000-0001-76374672; Email: makk.peter@ttk.bme.hu

Szabolcs Csonka - Department of Physics and Nanoelectronics "Momentum" Research Group of the Hungarian Academy of Sciences, Budapest University of Technology and Economics, 1111 Budapest, Hungary; Email: csonka.szabolcs@ttk.bme.hu

\section{Authors}

Tosson Elalaily - Department of Physics and Nanoelectronics "Momentum" Research Group of the Hungarian Academy of Sciences, Budapest University of Technology and Economics, 1111 Budapest, Hungary; Department of Physics, Faculty of Science, Tanta University, 31527 Tanta, Gharbia, Egypt; (1) orcid.org/0000-0003-3595-4798

Olivér Kürtössy - Department of Physics and Nanoelectronics "Momentum" Research Group of the Hungarian Academy of Sciences, Budapest University of Technology and Economics, 1111 Budapest, Hungary; (i) orcid.org/0000-0003-0221403X

Zoltán Scherübl - Department of Physics and Nanoelectronics "Momentum" Research Group of the Hungarian Academy of Sciences, Budapest University of Technology and Economics, 1111 Budapest, Hungary; Université Grenoble Alpes, CEA, Grenoble INP, IRIG, PHELIQS, 38000 Grenoble, France 
Martin Berke - Department of Physics and Nanoelectronics "Momentum" Research Group of the Hungarian Academy of Sciences, Budapest University of Technology and Economics, 1111 Budapest, Hungary

Gergö Fülöp - Department of Physics and Nanoelectronics "Momentum" Research Group of the Hungarian Academy of Sciences, Budapest University of Technology and Economics, 1111 Budapest, Hungary

István Endre Lukács - Center for Energy Research, Institute of Technical Physics and Material Science, H-1121 Budapest, Hungary

Thomas Kanne - Center for Quantum Devices and NanoScience Center, Niels Bohr Institute, University of Copenhagen, DK-2100 Copenhagen, Denmark

Jesper Nygård - Center for Quantum Devices and NanoScience Center, Niels Bohr Institute, University of Copenhagen, DK-2100 Copenhagen, Denmark

Kenji Watanabe - Research Center for Functional Materials, National Institute for Material Science, Tsukuba 305-0044, Japan; (1) orcid.org/0000-0003-3701-8119

Takashi Taniguchi - International Center for Materials Nanoarchitectonics, National Institute for Materials Science, Tsukuba 305-0044, Japan; 이이.org/0000-0002-14673105

Complete contact information is available at: https://pubs.acs.org/10.1021/acs.nanolett.1c03493

\section{Author Contributions}

T.E. and O.K. contributed equally to this work. T.E., O.K., and I.E.L. fabricated the devices, T.E., O.K., Z.S., M.B. and G.F. performed the measurements and did the data analysis. T.K. and J.N. grew the wires. K.W. and T.T. provided the highquality hBN. All authors discussed the results and worked on the manuscript. P.M. and Sz.Cs. guided the project.

\section{Notes}

The authors declare no competing financial interest.

\section{ACKNOWLEDGMENTS}

This work has received funding from Topograph FlagERA, the SuperTop QuantERA network, SuperGate Fet Open, and the FET Open AndQC, and from the OTKA FK-123894 grants. This research was supported by the Ministry of Innovation and Technology and the National Research, Development and Innovation Office within the Quantum Information National Laboratory of Hungary and by the Quantum Technology National Excellence Program (Project No. 2017-1.2.1-NKP2017-00001), by the UNKP-20-5 New National Excellence Program, the János Bolyai Research Scholarship of the Hungarian Academy of Sciences, by the Carlsberg Foundation, and by the Danish National Research Foundation. K.W. and T.T. acknowledge support from the Elemental Strategy Initiative conducted by the MEXT, Japan, Grant No. JPMXP0112101001, and JSPS KAKENHI, Grant Nos. $19 \mathrm{H} 05790$ and JP20H00354.

\section{REFERENCES}

(1) Devoret, M. H.; Wallraff, A.; Martinis, J. M. Superconducting qubits: A short review. arXiv Preprint (Condensed Matter: Mesoscale and Nanoscale Physics), 2004. https://arxiv.org/abs/cond-mat/ 0411174 (accessed 2021-02-13).

(2) Krantz, P.; Kjaergaard, M.; Yan, F.; Orlando, T. P.; Gustavsson, S.; Oliver, W. D. A quantum engineeras guide to superconducting qubits. Appl. Phys. Rev. 2019, 6, 021318.
(3) Wang, Z.; Miki, S.; Fujiwara, M. Superconducting nanowire single-photon detectors for quantum information and communications. IEEE J. Sel. Top. Quantum Electron. 2009, 15, 1741-1747.

(4) Natarajan, C. M.; Tanner, M. G.; Hadfield, R. H. Superconducting nanowire single-photon detectors: physics and applications. Supercond. Sci. Technol. 2012, 25, 063001.

(5) Aumentado, J. Superconducting parametric amplifiers: The state of the art in Josephson parametric amplifiers. IEEE Microwave Magazine 2020, 21, 45-59.

(6) Giazotto, F.; Martínez-Pérez, M. J. The Josephson heat interferometer. Nature 2012, 492, 401-405.

(7) Fornieri, A.; Giazotto, F. Towards phase-coherent caloritronics in superconducting circuits. Nat. Nanotechnol. 2017, 12, 944.

(8) Cleuziou, J.-P.; Wernsdorfer, W.; Bouchiat, V.; Ondarçuhu, T.; Monthioux, M. Carbon nanotube superconducting quantum interference device. Nat. Nanotechnol. 2006, 1, 53-59.

(9) Luomahaara, J.; Vesterinen, V.; Grönberg, L.; Hassel, J. Kinetic inductance magnetometer. Nat. Commun. 2014, 5, 4872.

(10) Soloviev, I. I.; Klenov, N. V.; Bakurskiy, S. V.; Kupriyanov, M. Y.; Gudkov, A. L.; Sidorenko, A. S. Beyond Mooreas technologies: operation principles of a superconductor alternative. Beilstein J. Nanotechnol. 2017, 8, 2689-2710.

(11) Frasca, S.; Charbon, E. Hybrid superconductor-semiconductor electronics. Nature Electronics 2019, 2, 433-434.

(12) De Simoni, G.; Paolucci, F.; Solinas, P.; Strambini, E.; Giazotto, F. Metallic supercurrent field-effect transistor. Nat. Nanotechnol. 2018, $13,802-805$.

(13) De Simoni, G.; Paolucci, F.; Puglia, C.; Giazotto, F. Josephson Field-Effect Transistors Based on All-Metallic $\mathrm{Al} / \mathrm{Cu} / \mathrm{Al}$ Proximity Nanojunctions. ACS Nano 2019, 13, 7871-7876.

(14) Paolucci, F.; De Simoni, G.; Solinas, P.; Strambini, E.; Puglia, C.; Ligato, N.; Giazotto, F. Field-effect control of metallic superconducting systems. AVS Quantum Science 2019, 1, 016501.

(15) Paolucci, F.; De Simoni, G.; Solinas, P.; Strambini, E.; Ligato, N.; Virtanen, P.; Braggio, A.; Giazotto, F. Magnetotransport Experiments on Fully Metallic Superconducting Dayem-Bridge Field-Effect Transistors. Phys. Rev. Appl. 2019, 11, 024061.

(16) Paolucci, F.; Vischi, F.; De Simoni, G.; Guarcello, C.; Solinas, P.; Giazotto, F. Field-Effect Controllable Metallic Josephson Interferometer. Nano Lett. 2019, 19, 6263-6269.

(17) De Simoni, G.; Puglia, C.; Giazotto, F. Niobium Dayem nanobridge Josephson gate-controlled transistors. Appl. Phys. Lett. 2020, 116, 242601.

(18) Rocci, M.; De Simoni, G.; Puglia, C.; Esposti, D. D.; Strambini, E.; Zannier, V.; Sorba, L.; Giazotto, F. Gate-Controlled Suspended Titanium Nanobridge Supercurrent Transistor. ACS Nano 2020, 14, 12621-12628.

(19) Puglia, C.; De Simoni, G.; Giazotto, F. Electrostatic control of phase slips in Ti Josephson nanotransistors. Phys. Rev. Appl. 2020, 13, 054026.

(20) Puglia, C.; De Simoni, G.; Ligato, N.; Giazotto, F. Vanadium gate-controlled Josephson half-wave nanorectifier. Appl. Phys. Lett. 2020, 116, 252601.

(21) Puglia, C.; De Simoni, G.; Giazotto, F. Gate control of superconductivity in mesoscopic all-metallic devices. Materials 2021, $14,1243$.

(22) Alegria, L. D.; Bøttcher, C. G.; Saydjari, A. K.; Pierce, A. T.; Lee, S. H.; Harvey, S. P.; Vool, U.; Yacoby, A. High-energy quasiparticle injection into mesoscopic superconductors. Nat. Nanotechnol. 2021, 16, 404-408.

(23) Ritter, M.; Fuhrer, A.; Haxell, D.; Hart, S.; Gumann, P.; Riel, H.; Nichele, F. A superconducting switch actuated by injection of high-energy electrons. Nat. Commun. 2021, 12, 1266.

(24) Golokolenov, I.; Guthrie, A.; Kafanov, S.; Pashkin, Y. A.; Tsepelin, V. On the origin of the controversial electrostatic field effect in superconductors. Nat. Commun. 2021, 12, 2747.

(25) Mercaldo, M. T.; Giazotto, F.; Cuoco, M. Spectroscopic Signatures of Gate-Controlled Superconducting Phases. arXiv Preprint 
(Condensed Matter: Superconductivity), 2020. arXiv:2012.01490. https://arxiv.org/abs/2012.01490 (accessed 2020-12-29).

(26) Mercaldo, M. T.; Solinas, P.; Giazotto, F.; Cuoco, M. Electrically tunable superconductivity through surface orbital polarization. Phys. Rev. Appl. 2020, 14, 034041.

(27) Chirolli, L.; Cea, T.; Giazotto, F. Impact of electrostatic fields in layered crystalline BCS superconductors. Physical Review. Research 2021, 3, 023135.

(28) Solinas, P.; Amoretti, A.; Giazotto, F. Sauter-Schwinger Effect in a Bardeen-Cooper-Schrieffer Superconductor. Phys. Rev. Lett. 2021, $126,117001$.

(29) Krogstrup, P.; Ziino, N.; Chang, W.; Albrecht, S.; Madsen, M.; Johnson, E.; Nygård, J.; Marcus, C. M.; Jespersen, T. Epitaxy of semiconductor-superconductor nanowires. Nat. Mater. 2015, 14, 400-406.

(30) Prada, E.; San-Jose, P.; de Moor, M. W.; Geresdi, A.; Lee, E. J.; Klinovaja, J.; Loss, D.; Nygård, J.; Aguado, R.; Kouwenhoven, L. P. From Andreev to Majorana bound states in hybrid superconductorsemiconductor nanowires. Nature Reviews Physics 2020, 2, 575-594.

(31) Plugge, S.; Landau, L.; Sela, E.; Altland, A.; Flensberg, K.; Egger, R. Roadmap to Majorana surface codes. Phys. Rev. B: Condens. Matter Mater. Phys. 2016, 94, 174514.

(32) Larsen, T. W.; Petersson, K. D.; Kuemmeth, F.; Jespersen, T. S.; Krogstrup, P.; Nygård, J.; Marcus, C. M. Semiconductor-nanowirebased superconducting qubit. Phys. Rev. Lett. 2015, 115, 127001.

(33) Hays, M.; De Lange, G.; Serniak, K.; Van Woerkom, D.; Bouman, D.; Krogstrup, P.; Nygård, J.; Geresdi, A.; Devoret, M. Direct microwave measurement of andreev-bound-state dynamics in a semiconductor-nanowire josephson junction. Phys. Rev. Lett. 2018, 121,047001

(34) Britnell, L.; Gorbachev, R. V.; Jalil, R.; Belle, B. D.; Schedin, F.; Katsnelson, M. I.; Eaves, L.; Morozov, S. V.; Mayorov, A. S.; Peres, N. M.; Castro Neto, A. H.; Leist, J.; Geim, A. K.; Ponomarenko, L. A.; Novoselov, K. S. Electron tunneling through ultrathin boron nitride crystalline barriers. Nano Lett. 2012, 12, 1707-1710.

(35) Fu, W.; Makk, P.; Maurand, R.; Bräuninger, M.; Schönenberger, C. Large-scale fabrication of BN tunnel barriers for graphene spintronics. J. Appl. Phys. 2014, 116, 074306.

(36) Kamalakar, M. V.; Dankert, A.; Bergsten, J.; Ive, T.; Dash, S. P. Enhanced tunnel spin injection into graphene using chemical vapor deposited hexagonal boron nitride. Sci. Rep. 2015, 4, 6146.

(37) Kammhuber, J.; Cassidy, M. C.; Zhang, H.; Gül, O.; Pei, F.; De Moor, M. W.; Nijholt, B.; Watanabe, K.; Taniguchi, T.; Car, D.; Plissard, S. R.; Bakkers, E. P. A. M.; Kouwenhoven, L. P. Conductance quantization at zero magnetic field in InSb nanowires. Nano Lett. 2016, 16, 3482-3486.

(38) Bretheau, L.; Joel, I.; Wang, J.; Pisoni, R.; Watanabe, K.; Taniguchi, T.; Jarillo-Herrero, P. Tunnelling spectroscopy of Andreev states in graphene. Nat. Phys. 2017, 13, 756-760.

(39) Ritter, M.; Crescini, N.; Haxell, D.; Hinderling, M.; Riel, H.; Bruder, C.; Fuhrer, A.; Nichele, F. On the Role of Out-of-Equilibrium Phonons in Gated Superconducting Switches. arXiv Preprint (Condensed Matter: Superconductivity), 2021. arXiv:2106.01816. https://arxiv.org/abs/2106.01816 (accessed 2021-06-12).

(40) Ambegaokar, V.; Baratoff, A. Tunneling between superconductors. Phys. Rev. Lett. 1963, 10, 486.

(41) Joo, S.; Kim, J.-J.; Yoo, J.; Park, M.; Lee, K.; Gu, G.; Lee, J. Cooper Pair Density of Bi2Sr2CaCu2O8+ $\mathrm{x}$ in Atomic scale at $4.2 \mathrm{~K}$. Nano Lett. 2019, 19, 1112-1117.

(42) Senkpiel, J.; Dambach, S.; Etzkorn, M.; Drost, R.; Padurariu, C.; Kubala, B.; Belzig, W.; Yeyati, A. L.; Cuevas, J. C.; Ankerhold, J.; Ast, R.; Kern, K. Single channel Josephson effect in a high transmission atomic contact. Commun. Phys. 2020, 3, 131.

(43) Gross, F.; Chandrasekhar, B.; Einzel, D.; Andres, K.; Hirschfeld, P.; Ott, H.; Beuers, J.; Fisk, Z.; Smith, J. Anomalous temperature dependence of the magnetic field penetration depth in superconducting UBe 13. Z. Phys. B: Condens. Matter 1986, 64, 175-188.

(44) Senapati, K.; Blamire, M. G.; Barber, Z. H. Spin-filter Josephson junctions. Nat. Mater. 2011, 10, 849-852.
(45) Zhang, J.; Ding, Z.; Huang, K.; Tan, C.; Hillier, A. D.; Biswas, P. K.; MacLaughlin, D. E.; Shu, L. Broken time-reversal symmetry in superconducting Pr 1- x La x Pt 4 Ge 12. Phys. Rev. B: Condens. Matter Mater. Phys. 2019, 100, 024508.

(46) Basset, J.; Stanisavljević, O.; Kuzmanović, M.; Gabelli, J.; Quay, C.; Estève, J.; Aprili, M. Gate-Assisted Phase Fluctuations in AllMetallic Josephson Junctions. arXiv Preprint (Condensed Matter: Superconductivity), 2021. arXiv:2107.05375. https://arxiv.org/abs/ 2107.05375 (accessed 2021-07-23). 